\title{
Diversidad y análisis fitosociológico de malezas en un cultivo de musáceas del trópico ecuatoriano
}

\author{
Vera, A. D., Palacios, Z. M., Liuba, D. A., Suarez, C. C. y Mendoza, H. C.
}

\begin{abstract}
RESUMEN
Uno de los primeros procesos para el desarrollo de nuevas herramientas de control y manejo integrado de malezas, implica el conocimiento de la composición y diversidad de la flora que incide en la producción del cultivo. El conocimiento de las especies de malezas dominantes es la base de la planificación de estrategias de control idóneas, que determinan el manejo del cultivo. Este trabajo analiza la diversidad de malezas y determina la identificación de las especies predominantes dentro del ecosistema de la localidad de Quevedo (Ecuador). Los resultados señalan que en el área monitoreada se encontraron 55 especies, incluidas en 22 familias, destacándose las Compositae, Poaceae y Euphorbiaceae con el mayor número. El ecosistema presenta diversidad levemente alta, dominancia alta y riqueza específica media. El análisis funcional revela 43 especies dicotiledóneas y 12 monocotiledóneas. Entre las especies de malezas dominantes se encontraron a la "piñita" (Murdannia nudiflora (L.) Brenan) que presenta nocividad media, seguida de la "caminadora" (Rottboellia exaltata L.f.) con alta nocividad y "tridax" (Tridax procumbens L.) con nivel medio de nocividad. Ninguna especie tiene un valor de importancia superior al 28,2\% en el área monitoreada.
\end{abstract}

Palabras clave: Diversidad, especies de malezas, dominancia, índice de valor de importancia.

Vera, A. D., Palacios, Z. M., Liuba, D. A., Suarez, C. C. and Mendoza, H. C., 2018. Diversity and phytosociological analysis of weeds in a Musaceae crops from the ecuatorian tropics. Agriscientia 35 (2): 43-52

\section{SUMMARY}

One of the first steps for the development of new control tactics and integrated weed management, is to know the composition and diversity of the weed flora affecting the crop. Knowledge of the dominant weed species is the basis 
forplanning suitable control strategies and determine the management of the crop. This work analyzes the diversity of weeds and identifiesthose species predominant within the ecosystem of Quevedo (Ecuador). Results showed 55 weed species belonging to 22 families, with the Compositae, Poaceae and Euphorbiaceae families having the higher number of species in the monitored area. The ecosystem presented slightly high diversity, high dominance, and medium specific richness. The functional analysis revealed 43 dicotyledonous species and 12 monocotyledonous species. Among the dominant weed species, we found the "piñita" (Murdannia nudiflora (L.) Brenan) that presented medium agresiveness, followed by the "caminadora" (Rottboellia exaltata L. f.) with high agresiveness and "tridax" (Tridax procumbens L.) with slight to medium level of agresiveness. No species had an importance value greater than $28.2 \%$ in the monitored area.

Keywords: Diversity, weed species, dominance, importance value index.

Vera, A. D., Palacios, Z. M., Liuba, D. A., Suarez, C. C. y Mendoza, H. C.: Facultad de Ciencias Agrarias, Universidad Técnica Estatal de Quevedo. Av. Quito, km 1.5, vía Santo Domingo de los Tsáchilas. Quevedo, Los Ríos, Ecuador. Correspondencia a: dvera@uteq.edu.ec

\section{INTRODUCCIÓN}

Las malas hierbas en muchos países son algunos de los principales obstáculos en la producción agrícola, ya que causan mermas en los cultivos y cuantiosas pérdidas económicas para las personas y sus intereses (Adkins y Shabbir, 2014). En los cultivos herbáceos y de hortalizas tales como la remolacha y las zanahorias, las pérdidas de rendimiento causadas por las malas hierbas pueden ser de hasta el $94 \%$ y $95 \%$ respectivamente (Coelho, Bianco y Carvalho, 2009; Bachega, Carvalho, Bianco y Cecilio, 2013). En zanahorias, si no hay control de malezas, las pérdidas pueden llegar al $94 \%$ (Coelho et al. 2009), mientras que en el caso de la remolacha entre un 70 a un $90 \%$ (Carvalho, Pitell, Cecilio, Bianco y Guzzo, 2008).

Es importante señalar que la interferencia no abarca todo el ciclo del cultivo y que el control de las malas hierbas debe llevarse a cabo durante el período crítico, es decir, durante el período de prevención total de la interferencia (PTPI) de estas plantas en el cultivo (Yirefu, Tana, Tafesse y Zekarias, 2012; Manpree, Makhan y Bhagirath 2014; Tursun, Datta, Tuncel y Knezevic, 2015; Matloob, et al., 2015), también teniendo en cuenta la estructura de la comunidad, representada por las especies presentes, su densidad y distribución.
Para entender la dinámica de las malas hierbas que viven en los cultivos en diferentes etapas de crecimiento es necesario identificarlas, porque no todas las especies son igualmente importantes en la interferencia que imponen al cultivo. Cada especie tiene el potencial para establecerse en un cultivo o zona y su interferencia puede actuar de forma diferente entre las plantas cultivadas (Cruz, Silva, Oliveira, Arcanjo y Alves, 2009).

Estudios fitosociológicos evalúan la comunidad de malezas por medio de índices que identifican las especies más importantes, para planificar las prácticas de gestión con el fin de facilitar cambios en los sistemas de control (Marqués, et al., 2011). Investigaciones realizadas por Johanns y Contiero (2006), manifiestan que las malas hierbas asociadas a los cultivos pueden estar relacionadas con las peculiaridades de cada región, en especial las especies predominantes.

El Litoral ecuatoriano posee condiciones óptimas de clima y suelos que favorecen el desarrollo de malezas como coquito (Cyperus rotundus L.), saboya (Panicum maximun Jacq.), la caminadora (Rottboellia exaltata L.f.), y otras que progresan de manera agresiva, vigorosa, dificultando el control y uso de los suelos (Palacios, 2016). Los pequeños agricultores consumen más del $40 \%$ de su tiempo laboral en operaciones de control manual de malezas y aun así sufren graves pérdidas debido a la competencia de estas. 
Las malezas perennes, especialmente gramíneas y ciperáceas, son un problema en muchas regiones del mundo, y Ecuador no es una excepción en el uso de herbicidas como principal herramienta de control. Los herbicidas postemergentes paraquat y glifosato se usan ampliamente. Paraquat defolia virtualmente a todas las especies de malezas, pero el glifosato es más útil donde las especies perennes, especialmente gramíneas y ciperáceas, son un problema.

En los sistemas de producción predominantes en la zona, el control está basado casi exclusivamente en el uso frecuente de herbicidas. Sin embargo, la eficiencia de este control es cuestionable debido a la metodología por la cual se selecciona el herbicida (Palacios, 2016). El objetivo de este trabajo es identificar y determinar las principales especies de malezas en un sistema agrícola de musáceas de la localidad de Quevedo (Ecuador).

\section{MATERIALES Y MÉTODOS}

La presente investigación se llevó a cabo durante el mes de junio del 2015, en la localidad de Quevedo, provincia de Los Ríos, Ecuador, entre las coordenadas geográficas $79^{\circ} 27^{\prime} \mathrm{O}, 1^{\circ} 06^{\prime}$ S, a 120 m s.n.m. El clima de la zona es tropical húmedo, la temperatura media anual es $25,9^{\circ} \mathrm{C}$, la precipitación promedio anual es 3019,6 mm, la humedad relativa es $83 \%$ y la heliofanía promedio anual es 1030,6 horas (Instituto Nacional de Meteorología e Hidrología, 2015). El suelo es de textura franco-arcillosa, con $\mathrm{pH}$ de 6,0 y drenaje regular.

El sistema agrícola monitoreado fue una plantación mixta de cultivares con mayor potencial de aceptación por parte de los productores: la variedad predominante local Barraganete se combinó con cuatro cultivares (Orito, Limeño, Maqueño y Gros Michel). La plantación perenne de musáceas tenía dos años, se encontraba en período de cosecha todo el año, con una densidad de siembra de 1111 plantas ha-1. Este sistema es representativo de la zona central del Litoral tanto en cuanto al tipo de explotación agrícola, como al tipo de suelo. El campo relevado estuvo dedicado por más de 30 años a cultivos herbáceos intensivos (maíz, arroz y soja) y tiene una pendiente de $2 \%$. Estas condiciones son comunes en las explotaciones del área.

El manejo de malezas del cultivo se basa principalmente en controles mecanizados con motoguadaña, durante todo el año. Dos veces al año, cuando la población de malas hierbas dificulta el control mecánico, se realiza la aplicación de 2,5
I ha ${ }^{-1}$ de glifosato con la finalidad de mantener las malezas bajo control sin que causen problemas al cultivo.

El área monitoreada estuvo representada por una hectárea donde se demarcaron 22 puntos de monitoreo, en los cuales se realizaron 12 submonitoreos dando un total de 264 cuadrantes de $0,33 \mathrm{~m} \times 1 \mathrm{~m}$, siguiendo la metodología descrita por Muller-Dombois y Ellenberg (1974). En ella se determinó la lista de especies y su abundancia. Los datos obtenidos se extrapolaron al $\mathrm{m}^{2}$ obteniendo de este modo el número de individuos por $\mathrm{m}^{2}$. La identificación de las especies y funciones se realizó utilizando bibliografía específica de Ordeñana (1992), Toro y Briones (1984), Gómez y Rivero (1987), Cardenas, Reyes y Doll (1972) y Mejía, (2010).

\section{Determinación de la diversidad existente}

Para la determinación de los parámetros de diversidad en cada cuadrante demarcado se cuantificó la riqueza de especies y la abundancia de cada especie de malezas. Los índices de diversidad local o alfa $(\alpha)$ se calcularon de acuerdo a los utilizados por Moreno (2001):

Índice de Margalef (riqueza)

$$
D_{M g}=\frac{(S-1)}{N}
$$

Donde

$S=$ número de especies

$N=$ número total de individuos

Valores inferiores a 2 son considerados como relacionados con zonas de baja biodiversidad (en general resultado de efectos antropogénicos) y valores superiores a 5 son considerados como indicativos de alta biodiversidad (Margalef, 1969).

\section{Índice Simpson 1-D (dominancia)}

$$
\lambda=P_{i}^{2}
$$

\section{Donde}

$\mathrm{P}_{\mathrm{i}}=$ abundancia proporcional de la especie $i$, lo cual implica obtener el número de individuos de la especie $i$ dividido entre el número total de individuos de la muestra.

Tomando en cuenta que varía de 0 a 1 , y mide la probabilidad de que dos individuos extraídos al azar pertenezcan a una misma especie, para este índice el 1 indica que no hay diversidad y que la dominancia es alta (Lamprecht, 1990). 
Índice Shannon (abundancia)

$$
H^{\prime}=P_{i} \in P_{i}
$$

Puede adquirir valores entre 0 , cuando hay una sola especie es decir que la diversidad es baja, y excepcionalmente puede haber ecosistemas que superen el valor de 5. La mayor limitante de este índice es que no tiene en cuenta la distribución de las especies en el espacio, puede verse fuertemente influenciado por las especies más abundantes (Moreno, 2001).

Índice de valor de importancia

Para el cálculo del índice de valor de importancia (IVI), se aplicó la ecuación de Curtis \& Mcintosh, (1951)

$$
\begin{aligned}
& I V I=A r+D r+F r \\
& \text { Donde } \\
& \mathrm{Ar}=\text { Abundancia relativa } \\
& \mathrm{Dr}=\text { Dominancia relativa } \\
& \mathrm{Fr}=\text { Frecuencia relativa }
\end{aligned}
$$

\section{Abundancia de las especies de malezas}

Este parámetro se calculó empleando la formula siguiente:

Abundancia $(A)=$ Valor obtenido por la especie en los cuadros (Vsi) Número total de cuadros con la especie (Cs)

\section{Dominancia de las especies de malezas}

Para registrar este parámetro, se evaluó la cantidad de malezas presentes por $\mathrm{m}^{2}$ en cada tratamiento. Esta evaluación se realizó 15 días antes de la aplicación utilizando para el efecto la siguiente fórmula:

Dominancia $(D)=$ Número de individuos de la especie Unidad de superficie muestreada

\section{Frecuencia de las especies de malezas}

Este valor fue establecido utilizando la siguiente fórmula:

Frecuencia $(F)=$ Número de cuadros con la especie(cs)

Número total de cuadros $(\mathrm{ct}) \times 100$

Finalmente, de acuerdo a la metodología de Muller-Dombois y Ellenberg (1974), se realizó la clasificación fitosociológica, considerando la clase de presencia que se indica en Tabla 1. Los datos de diversidad fueron analizados utilizando el software estadístico Past3 (Hammer, 2001).

\section{RESULTADOSY DISCUSIÓN}

\section{Determinación de la diversidad de malezas existentes}

La lista de especies (Tabla 2), revela que dentro del agroecosistema monitoreado se encontraron 55 especies de malezas asociadas al lugar de estudio, las cuales representan 22 familias diferentes. Espinoza (2008), en un estudio realizado en tres localidades de la provincia utilizando la misma metodología, registró 17 familias de malezas. Vera (2008), en un área cercana a la localización del presente estudio, encontró 23 especies de malezas en sistemas de monocultivo de plátano, cacao y sus asociados.

Tabla 2. Índices de diversidad de malezas

\begin{tabular}{lc}
\hline Familias & 22 \\
Riqueza & 55 \\
Simpson_1-D & 0,93 \\
Shannon_H & 3,22 \\
\hline
\end{tabular}

El índice de Simpson $(0,93)$ indica que la diversidad es baja y que la dominancia es alta,

Tabla 1. Clasificación fitosociológica de las comunidades vegetales y de sus relaciones con el medio de acuerdo a Muller y Ellenberg, 1979.

\begin{tabular}{ll}
\multicolumn{1}{c}{$\begin{array}{c}\text { Clase de } \\
\text { presencia }\end{array}$} & \multicolumn{1}{c}{ Valor de abundancia - dominancia } \\
\hline r: $<6 \%$ & r: Individuos raros o aislados \\
$+: 7-10 \%$ & $+:$ Individuos poco abundantes, de débil cobertura \\
I: $11-20 \%$ & 1: Individuos bastante abundantes, pero con débil cobertura \\
II: $21-40 \%$ & 2: Individuos muy abundantes que cubren $1 / 20(5-25 \%)$ de la superficie \\
III: $41-60 \%$ & 3: Individuos de número variable, que cubren de $1 / 4$ a $1 / 2(25-50 \%)$ de la superficie \\
IV: $61-80 \%$ & 4: Individuos de número variable, que cubren de $1 / 2$ a $3 / 4(50-75 \%)$ de la superficie \\
V: $>81 \%$ & 5: Individuos de número variable, que cubren más de $3 / 4(>75 \%)$ de la superficie \\
\hline
\end{tabular}


existiendo una alta probabilidad de que dos individuos extraídos al azar pertenezcan a una misma especie. Este valor se diferencia del índice promedio de la zona reportado por Espinoza (2008), que muestra baja dominancia y alta diversidad.

El índice de Shannon $(3,22)$ muestra una diversidad levementealta, indicando la probabilidad de encontrar un determinado individuo en el área del ecosistema monitoreado, teniendo en cuenta la uniformidad de la distribución de las especies en el espacio muestreado. Esto se diferencia levemente del índice de la zona presentado por Espinoza (2008), correspondiente a 2,34, y diversidad herbácea de 2,08 en la provincia. Al medir la riqueza específica (Margalef), se pudo apreciar una diversidad alta $(5,38)$.

El análisis funcional de las características reproductivas e interferencia de la comunidad de malezas basado en la caracterización de Ordeñana (1992); Toro et al. (1984), Gómez y Rivero (1987), Cárdenas et al. (1972) y Mejía (2010) (Tabla 3), muestra que dentro de los 55 morfotipos (especies) se encontraron 43 dicotiledóneas y 12 monocotiledóneas. El elenco incluye la piñita (Murdannia nudiflora (L.) Brenan) y la caminadora (Rottboellia exaltata L.f.) consideradas como especies de importancia dentro del área muestreada, debido a su vigor y amplia competencia con los cultivos que las torna problemáticas e invasoras de la región (Labrada, 2004).

La relación de interferencia es de 3,58 especies dicotiledóneas por cada monocotiledónea. De las especies encontradas 42 son de ciclo anual, 11 perennes y 2 reportadas como anuales y perennes. En cuanto a la propagación, 46 especies presentaron reproducción por semillas, 1 especie reproducción vegetativa y 7 reproducción tanto vegetativa como por semillas.

La evaluación y monitoreo de comunidades de malezas durante y después de cada ciclo de cultivo, debe transcender el análisis de parámetros fitosociológicos de grupos funcionales, para considerar también un enfoque que integre la importancia relativa de las especies en relación a los rasgos que permiten su supervivencia y dominancia en la comunidad. Estudios recientes han logrado identificar y explicar rasgos funcionales de malezas asociados con el efecto de las perturbaciones inducidas por diferentes prácticas de manejo agrícolas y gradientes de recursos (Perronne, Le Corre, Bretagnolle y Gaba, 2015; Cordeau, Ryan, Bohan, Reboud y Chauvel, 2017; Gaba, et al., 2017).
El mayor número de especies encontradas dentro del área monitoreada pertenece a la familia Asteracea (10 especies), seguida de las familias Poaceae y Euphorbiaceae (8 especies para cada familia). Además, se encontraron representantes de las familias Convolvulaceae, Solanaceae, Fabaceae y Verbenaceae con tres especies cada una, la familia Commelinaceae y Cyperaceae con dos especies, las restantes 13 especies, entre las que se encuentran las familias Capparidaceae y Cucurbitaceae con una especie cada una (Tabla 3). Espinoza (2008), menciona 15 familias y dos subfamilias de malezas registradas, de las cuales siete familias coinciden con las mencionadas en esta investigación.

\section{Composición de las especies de malezas e índice de valor de importancia (IVI)}

Entre las especies que se encuentran en la clase de presencia II, que considera una cobertura entre el 5 y el $25 \%$ de la superficie muestreada, se encontró a la piñita (M. nudiflora) (Tabla 4), siendo inferior al índice del $53 \%$ reportado por Escobar (2007), donde se evidencia fitosociológicamente que esta especie se encuentra en la clase de presencia III que considera una cobertura entre el 25 y $50 \%$ de la superficie muestreada. Vera (2008), en estudios similares en la zona, encontró como malezas dominantes a la cristalina (Peperomia pellucida L. H.B.K.) con un IVI entre 11,16 y $19,58 \%$ en un sistema asociado (cacao y plátano) y en monocultivo de plátano; y a la hierba de puerco (Galinsoga sp.) con un IVI de 13,75\% en un monocultivo de cacao, lo que determina que el cultivo influye sobre las especies de malezas presentes.

La caminadora ( $R$. exaltata) es la segunda especie de maleza en importancia dentro de los agroecosistemas monitoreados. Ésta se ha tornado dominante, superando el valor encontrado por Vera (2008) en varios sistemas de cultivos, en los cuales no sobrepasa el IVI de 0,91\%, pero inferior al índice presentado por Escobar (2007), con el $53 \%$. La caminadora ( $R$. exaltata) es una especie considerada exótica. La velocidad con la cual logra incorporarse a la flora existente causa problemas en los cultivos. Una especie puede mantener bajo nivel poblacional al introducirse en un sistema productivo o ecosistema, y su expansión estará sujeta a encontrar un sitio seguro dentro del alcance de su dispersión (Labrada, 2004).

En la clase de presencia I se ubican las especies abundantes pero con débil cobertura, entre las que se encuentran tridax (Tridax procumbens 
Tabla 3. Características reproductivas e interferencia de las especies de malezas.

\begin{tabular}{|c|c|c|c|c|c|c|}
\hline \multicolumn{2}{|r|}{ MALEZAS } & \multirow{2}{*}{ Ciclo } & \multirow{2}{*}{$\begin{array}{c}\text { Morfo- } \\
\text { tipo }\end{array}$} & \multirow{2}{*}{ Propagación } & \multirow{2}{*}{ Familia } & \multirow{2}{*}{$\begin{array}{l}\text { Grado } \\
\text { nocivo }\end{array}$} \\
\hline Nombre común & Nombre científico & & & & & \\
\hline Piñita & Murdannia nudiflora (L.) Brenan & A & Mono & $\mathrm{S} / \mathrm{V}$ & Commelinaceae & $\mathrm{Al}$ a $\mathrm{M}$ \\
\hline Caminadora & Rottboellia exaltata L.f. & A & Mono & S & Poaceae & $\mathrm{Al}$ \\
\hline Tridax & Tridax procumbens L. & $A$ & Dico & S & Compositae & $\mathrm{M}$ a L \\
\hline Cerbatana & Synedrella nodiflora (L.) Gaertn & $A$ & Dico & S & Compositae & M \\
\hline Platanito & Cleome affinis DC & A & Dico & S & Capparidaceae & M \\
\hline Venadillo & Conyza bonariensis (L.) Cronq. & $\mathrm{P}$ & Dico & S & Compositae & --- \\
\hline Hierba de chivo & Ageratum conyzoides L. & $A$ & Dico & S & Compositae & M \\
\hline Guarda roció & Digitaria sanguinalis L. Scop. & A & Mono & $\mathrm{S} / \mathrm{V}$ & Poaceae & M \\
\hline Moradita & Vernonia cinerea (L.) Less & $A$ & Dico & S & Asteraceae & M \\
\hline Pega pega & Desmodium incanum (SW.) DC & $P$ & Dico & S & Papilionaceae & \\
\hline Falsa verbena & Pseudelephantopus spicatus (Aubl.) Gleas & $P$ & Dico & $S$ & Compositae & -- \\
\hline Tripa de pollo & Euphorbia hirta L. & A & Dico & S & Euphorbiaceae & M \\
\hline Lechosa & Euphorbia heterophylla L. & A & Dico & S & Euphorbiaceae & $\mathrm{Al}$ a M \\
\hline Paraguay & Lindernia crustacea (L.) F. Muell & $P$ & Dico & S & Scrophulariaceae & $\mathrm{COB}$ \\
\hline Clavo de agua & Ludwigia erecta (L.) Hara & A & Dico & S & Onagraceae & M \\
\hline Cristalina & Peperomia pellucida L. H.B.K. & A & Dico & S & Piperaceae & $\mathrm{L}$ \\
\hline Escoba negra & Sida rhombifolia L. & $P$ & Dico & S & Malvaceae & M \\
\hline Paja blanca & Chloris polydactyla L. Swartz & A & Mono & S & Poaceae & $\mathrm{L}$ \\
\hline Arrocillo & Fimbristylis dichotoma L. Vahl. & $A$ & Mono & S & Cyperaceae & M \\
\hline Falso johnson & Sorghum verticilliflorum (Steud) Stapf. & A & Mono & S & Poaceae & M \\
\hline Ortigilla & Fleurya aestuans L. Guard & $A$ & Dico & $S$ & Urticaceae & M \\
\hline Caperonia & Caperonia palustris St. Hil. & $A$ & Dico & S & Euphorbiaceae & M \\
\hline Balsilla & Phyllanthus niruri L. & A & Dico & S & Euphorbiaceae & M \\
\hline Rabo de alacrán & Cyathula prostrata (L.) Blume. & $A$ & Dico & $S$ & Amaranthaceae & M \\
\hline Balsilla & Phyllanthus urinaria Wall. & A & Dico & S & Euphorbiaceae & --- \\
\hline Gusanillo & Acalypha alopecuroides Jacq. & $A$ & Dico & S & Euphorbiaceae & $L$ \\
\hline Paja de burro & Eleusine indicaL. Gaertn. & $A$ & Mono & $S$ & Poaceae & M \\
\hline Escoba negra & Sida acuta Burm. f. & $A / P$ & Dico & S & Malvaceae & M \\
\hline Betilla & Ipomoea sp. & $A$ & Dico & S & Convolvulaceae & $\mathrm{Al} / \mathrm{M} / \mathrm{L}$ \\
\hline Saboya & Panicum maximum Jacq. & $P$ & Mono & V & Poaceae & M \\
\hline Cyperáceas & Cyperus sp. & $P$ & Mono & $\mathrm{S} / \mathrm{V}$ & Cyperaceae & $\mathrm{AL} / \mathrm{M}$ \\
\hline Duraznillo blanco & Solanum glaucophyllum Desf. & $P$ & Dico & S & Solanaceae & --- \\
\hline Teatina & Scoparia dulcis L. & A & Dico & S & Plantaginaceae & --- \\
\hline Uvilla & Cissus sicyoides L. & $P$ & Dico & $\mathrm{S} / \mathrm{V}$ & Vitaceae & M \\
\hline Frijol de monte & Phaseolus lathyroides L. & $A$ & Dico & S & Leguminosae & M \\
\hline Hierba de toro & Cuphea racemosa(L.) Spreng. & $A$ & Dico & S & Lythraceae & M \\
\hline Frijolillo & $\begin{array}{c}\text { Vigna peduncularis (Kunth.) Feweet \& } \\
\text { Rendle }\end{array}$ & $A$ & Dico & $\mathrm{S} / \mathrm{V}$ & Leguminosae & $\mathrm{COB}$ \\
\hline Hierba mora & Solanum nigrum Sendt. & A & Dico & S & Solanaceae & M \\
\hline Mongona & Commelina diffusa Burm. f. & $A / P$ & Mono & S.V & Commelinaceae & M \\
\hline Bejuquillo & Teramnus volubilis Sw. & $A$ & Dico & S & Leguminosae & $\mathrm{COB}$ \\
\hline Drimaria & Drymaria cordata L. Willd. & A & Dico & S & Caryophyllaceae & $\mathrm{M}$ aCOB \\
\hline Amor seco & Bidens pilosa L. & $A$ & Dico & S & Compositae & M \\
\hline Pincel & Emilia sonchifolia L. DC. & A & Dico & S & Compositae & $L$ \\
\hline Tomillo & Mollugo verticillata $\mathrm{L}$. & A & Dico & S & Aizoaceae & $\mathrm{L}$ \\
\hline Verbena negra & Stachytarpheta cayennensis (L.C.Rich.) Vahl. & $\mathrm{A} / \mathrm{P}$ & Dico & S & Verbenaceae & M \\
\hline Paja brava & Paspalum paniculatum L. & $\mathrm{P}$ & Mono & $\mathrm{S} / \mathrm{V}$ & Poaceae & M \\
\hline Mastrantillo & Hyptis mutabilis (Rich.) Briq. & $A$ & Dico & S & Lamiaceae & --- \\
\hline Cadillo de bolsa & Priva lappulacea (L.) Pers. & $P$ & Dico & S & Verbenaceae & M \\
\hline Lecherito & Euphorbia hypericifolia L. & $A$ & Dico & $S$ & Euphorbiaceae & M \\
\hline Estrellita de belén & Ipomoea quamoclit L. & $A$ & Dico & S & Convolvulaceae & $\mathrm{L}$ \\
\hline
\end{tabular}


(Cont. tabla 3)

Botón de oro

Achochilla

Botoncillo blanco

Paja de patillo

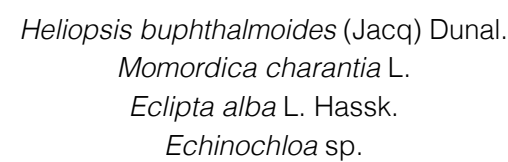

Heliopsis buphthalmoides (Jacq) Dunal Momordica charantia L. Eclipta alba L. Hassk. Echinochloa sp.

Vejigón Physalis angulata $\mathrm{L}$.

$\begin{array}{ll}\text { A } & \text { Dico } \\ \text { A } & \text { Dico } \\ \text { A } & \text { Dico } \\ \text { A } & \text { Mono } \\ \text { A } & \text { Dico }\end{array}$

\begin{tabular}{lcc} 
S & Compositae & --- \\
S & Cucurbitaceae & $\mathrm{M}$ \\
$\mathrm{S}$ & Compositae & $\mathrm{M}$ \\
$\mathrm{S}$ & Poaceae & $\mathrm{Al} / \mathrm{M}$ \\
$\mathrm{S}$ & Solanaceae & $\mathrm{M}$ \\
\hline
\end{tabular}

$\mathrm{A}=$ Anual, $\mathrm{P}=$ Perenne, Mono= Monocotiledónea, Dico= Dicotiledónea, $\mathrm{S}=$ Semilla, $\mathrm{V}=$ Vegetativa, $\mathrm{Al}=$ Altamente nocivo, $\mathrm{M}=$ Medianamente nocivo, $L=$ Levemente nocivo.

Tabla 4. Especies de malezas, índice de valor de importancia IVI (\%) y clase de presencia de malezas

\begin{tabular}{|c|c|c|c|c|c|c|c|c|c|}
\hline \multicolumn{2}{|r|}{ MALEZAS } & \multirow{2}{*}{ D } & \multirow{2}{*}{$\begin{array}{l}\text { DR } \\
(\%)\end{array}$} & \multirow{2}{*}{$\mathbf{F}$} & \multirow{2}{*}{$\begin{array}{l}\text { FR } \\
(\%)\end{array}$} & \multirow{2}{*}{ A } & \multirow{2}{*}{ AR } & \multirow{2}{*}{$\begin{array}{l}\text { IVI } \\
\text { (\%) }\end{array}$} & \multirow{2}{*}{$\mathrm{CP}$} \\
\hline Nombre común & Nombre científico & & & & & & & & \\
\hline Piñita & Murdannia nudiflora (L.) Brenan & 13,30 & 15,47 & 0,58 & 4,49 & 23,03 & 8,26 & 28,23 & II \\
\hline Caminadora & Rottboellia exaltata L.f. & 12,42 & 14,45 & 0,75 & 5,87 & 16,45 & 5,90 & 26,22 & II \\
\hline Tridax & Tridax procumbens $\mathrm{L}$. & 7,57 & 8,81 & 0,66 & 5,11 & 11,53 & 4,14 & 18,05 & $\mathrm{I}$ \\
\hline Cerbatana & Synedrella nodiflora (L.) Gaertn & 4,04 & 4,70 & 0,46 & 3,55 & 8,84 & 3,17 & 11,42 & I \\
\hline Platanito & Cleome affinis DC & 3,75 & 4,37 & 0,41 & 3,20 & 9,13 & 3,27 & 10,84 & I \\
\hline Venadillo & Conyza bonariensis (L.) Cronq. & 3,32 & 3,86 & 0,29 & 2,29 & 11,27 & 4,04 & 10,19 & $\dagger$ \\
\hline Hierba de chivo & Ageratum conyzoidesL. & 3,13 & 3,64 & 0,29 & 2,23 & 10,92 & 3,92 & 9,79 & $\dagger$ \\
\hline inarda rocio & Digitaria sanguinalis L. Scop. & & 3,23 & 0,62 & 4,82 & 4,48 & 1,61 & 9,65 & $\dagger$ \\
\hline Moradita & Vernonia cinerea (L.) Less & & 3,44 & 0,52 & 4,05 & 5,68 & 2,04 & 9,53 & $\dagger$ \\
\hline Pega pega & \multirow{3}{*}{$\begin{array}{l}\text { Desmodium incanum (SW.) DC } \\
\text { Pseudelephantopus spicatus (Aubl.) } \\
\text { Gleas } \\
\text { Euphorbia hirta L. }\end{array}$} & 2,94 & 3,42 & 0,49 & 3,85 & 5,95 & 2,14 & 9,41 & $\dagger$ \\
\hline Falsa verbena & & 1,77 & 2,06 & 0,16 & 1,26 & 10,91 & 3,91 & 7,23 & $\dagger$ \\
\hline Tripa de pollo & & 1,89 & 2,20 & 0,46 & 3,55 & 4,13 & 1,48 & 7,23 & $\dagger$ \\
\hline Lechosa & Euphorbia heterophylla L. & & 2,17 & 0,22 & 1,70 & 8,53 & 3,06 & 6,94 & $\dagger$ \\
\hline Paraguay & Lindernia crustac & & 2,16 & 0,38 & 2,94 & 4,93 & ,77 & 6,87 & $\dagger$ \\
\hline agua & Ludwigi & & 1,83 & 0,46 & 3,61 & 9 & 2 & 6,66 & $\dagger$ \\
\hline & Pep & & 1,9 & 0 , & & 5,6 & & 6,30 & $\mathrm{r}$ \\
\hline negra & Sida & & 1,46 & 0,48 & 3,73 & 2,62 & 0,94 & 6,13 & r \\
\hline lanca & Chlor & & 0,87 & 0,06 & 0,44 & 13,20 & 4,73 & 6,04 & r \\
\hline rrocillo & Fimbris & & 1,77 & 0,27 & 2,08 & & 2,04 & 5,89 & r \\
\hline also johnson & Sorghum verticilli & & 1,58 & 0,35 & 2,76 & 3,8 & 1,37 & 5,71 & r \\
\hline Ortigilla & Fleurya aestuans & 1,0 & 1,24 & 0,11 & 0,85 & 9,76 & 3,50 & 5,59 & r \\
\hline aperonia & Cape & & 1,33 & 0,37 & 2,91 & 3,05 & 1,09 & 5,33 & $r$ \\
\hline Balsilla & Phylla & 1,23 & 1,43 & 0,28 & 2,14 & 4,45 & 1,60 & 5,17 & $r$ \\
\hline abo de a & Cyathul & & 1,10 & 0,21 & 1,64 & 4,48 & 1,61 & 4,35 & $r$ \\
\hline Balsilla & Phyllanthus uri & & 1,05 & 0,17 & 1,35 & 5,22 & 1,87 & 4,28 & r \\
\hline Gusanillo & Acalypha alopecur & & 0,72 & & 1,00 & 4,79 & 1,7 & 3,43 & r \\
\hline Paja de & Eleus & & & & & & & 3,3 & $\mathrm{r}$ \\
\hline Escoba & Sida $\mathrm{a}$ & & & & & & & 3,35 & $\mathrm{r}$ \\
\hline Betilla & Ipom & & & & & & & 3,10 & r \\
\hline Saboya & aximum Jacq. & & 0,61 & 0,17 & 1,32 & 3,0 & 1,1 & 3,03 & r \\
\hline Cyper & Суре & & 0,5 & 0,22 & 1,73 & 2,0 & 0,73 & 2,99 & $r$ \\
\hline Duraznillo blanco & Solant & & 0,5 & 0,1 & 1,29 & 2,8 & 1,0 & 2,85 & r \\
\hline Teatina & Scop & & 0,54 & 0,1 & 1,35 & 2,65 & 0,95 & 2,84 & r \\
\hline Uvilla & icyoides L. & 0,45 & 0,52 & 0,17 & 1,32 & 2,64 & 0,95 & 2,79 & r \\
\hline Frijol de monte & Phaseolus lathyroides L. & & 0,44 & 0,08 & 0,62 & 4,76 & 1,71 & 2,76 & r \\
\hline Hierba de toro & \multirow{3}{*}{$\begin{array}{l}\text { Cuphea racemosa (L.) Spreng. } \\
\text { Vigna peduncularis (Kunth.) Feweet \& } \\
\text { Rendle } \\
\text { Solanum nigrum Sendt. }\end{array}$} & 0,42 & 0,49 & 0,11 & 0,85 & 3,83 & 1,37 & 2,71 & r \\
\hline Frijolillo & & 0,43 & 0,50 & 0,13 & 1,00 & 3,32 & 1,19 & 2,69 & $r$ \\
\hline Hierba mora & & 0,31 & 0,36 & 0,06 & 0,47 & 5,06 & 1,82 & 2,64 & r \\
\hline Mongona & Commelina diffusa Burm. f. & 0,38 & 0,45 & 0,17 & 1,35 & 2,22 & 0,80 & 2,59 & r \\
\hline & & 0,2 & 0,32 & 0,07 & 0,53 & 4,00 & 1,43 & 2,28 & $r$ \\
\hline & Drymaria cordata L. Willd. & 0,23 & 0,27 & 0,05 & 0,41 & 4,43 & 1,59 & 2,27 & $r$ \\
\hline Amor seco & Bidens pilosa $\mathrm{L}$. & 0,30 & 0,35 & 0,14 & 1,12 & 2,08 & 0,75 & 2,21 & $\mathrm{r}$ \\
\hline
\end{tabular}




\begin{tabular}{|c|c|c|c|c|c|c|c|c|}
\hline Pincel & Emilia sonchifolia L. DC. & 0,30 & 0,35 & 0,14 & 1,06 & 2,22 & 0,80 & 2,21 \\
\hline Tomillo & Mollugo verticillata $L$. & 0,29 & 0,34 & 0,13 & 1,03 & 2,23 & 0,80 & 2,17 \\
\hline Verbena negra & $\begin{array}{l}\text { Stachytarpheta cayennensis (L.C.Rich.) } \\
\text { Vahl. }\end{array}$ & 0,11 & 0,12 & 0,02 & 0,18 & 4,67 & 1,67 & 1,97 \\
\hline Paja brava & Paspalum paniculatum L. & 0,24 & 0,28 & 0,12 & 0,97 & 1,91 & 0,68 & 1,93 \\
\hline Mastrantillo & Hyptis mutabilis (Rich.) Briq. & 0,21 & 0,25 & 0,07 & 0,53 & 3,11 & 1,12 & 1,89 \\
\hline Cadillo de bolsa & Priva lappulacea (L.) Pers. & 0,18 & 0,21 & 0,06 & 0,50 & 2,76 & 0,99 & 1,70 \\
\hline Lecherito & Euphorbia hypericifolia L. & 0,19 & 0,22 & 0,08 & 0,62 & 2,38 & 0,85 & 1,69 \\
\hline Estrellita de belén & Ipomoea quamoclit L. & 0,12 & 0,14 & 0,09 & 0,68 & 1,35 & 0,48 & 1,29 \\
\hline Botón de oro & Heliopsis buphthalmoides ( Jacq) Dunal & 0,08 & 0,09 & 0,03 & 0,26 & 2,33 & 0,84 & 1,19 \\
\hline Achochilla & Momordica charantiaL. & 0,06 & 0,07 & 0,03 & 0,21 & 2,43 & 0,87 & 1,15 \\
\hline Botoncillo blanco & Eclipta alba L. Hassk. & 0,05 & 0,05 & 0,04 & 0,29 & 1,20 & 0,43 & 0,78 \\
\hline Paja de patillo & Echinochloa sp. & 0,02 & 0,03 & 0,02 & 0,18 & 1,00 & 0,36 & 0,56 \\
\hline Vejigón & Physalis angulata L. & 0,02 & 0,02 & 0,02 & 0,12 & 1,00 & 0,36 & 0,49 \\
\hline Total & & 85,95 & 100 & 12,9 & 100 & 279 & 100 & 300 \\
\hline
\end{tabular}

${ }^{*}$ DR: Densidad relativa; FR: Frecuencia relativa; AR: Abundancia relativa; IVI: Índice de valor de importancia; CP: Clase de presencia.

L.), cerbatana (Synedrella nodiflora (L.) Gaernt) y platanito (Cleome affinis DC), con porcentajes de IVI entre 18,05 y $10,84 \%$.

Las especies venadillo (Conyza bonariensis (L.) Cronq.), hierba de chivo (Ageratum conyzoides L.), guardarocío (Digitaria sanguinalis L. Scop.), moradita (Vernonia cinerea (L.) Less), pega pega (Desmodium incanum (SW.) DC), falsa verbena (Pseudelephantopus spicatus (Aubl.) Gleas), tripa de pollo (Euphorbia hirta L.), lechosa (E. heterophylla), paraguay (Lindernia crustacea (L.) F. Muell) y clavo de agua (Ludwigia erecta (L.) Hara), se ubican en la clase poco abundante y de poca cobertura $(\boldsymbol{+})$ con densidad relativamente baja comparado con los valores encontrados por Rodríguez (2013), quien encontró promedios de 29,88 individuos $\mathrm{m}^{-2}$ en potreros. Según el trabajo realizado por Venegas y Muñoz (1984), son malezas comunes en el Litoral, venadillo (Ageratum conyzoides L.), tripa de pollo (Euphorbia hirta L.) y escoba negra (Sida rhombifolia L.). Las 40 especies restantes se ubican en la clase $\mathbf{r}$ consideradas fitosociológicamente como especies raras o aisladas debido a encontrarse con porcentajes de abundancia inferior a $6,5 \%$, entre las que se encuentran cristalina ( $P$. pellucida) y vejigón (Physalis angulata L.). Según Venegas y Muñoz (1984), estas son malezas tropicales comunes en el Litoral del Ecuador.

Una situación de alta densidad de malezas puede cambiar de un punto de monitoreo a otro incluso estando contiguos. Esto coincide con lo comentado por Cardina, Regnier y Harrison (1991) respecto a que la composición y abundancia de especies cambian en respuesta al manejo del cultivo y del suelo.
El grado de nocividad de las malezas según la caracterización de clasificación de Ordeñana (1992), Toro et al. (1984), Gómez y Rivero (1987), Cardenas et al. (1972) y Mejía (2010) (Tabla 4) muestra que la caminadora ( $R$. exaltata) presenta una alta nocividad. Esta característica junto con su amplia adaptación y competencia con el cultivo hace de esta especie uno de los biotipos dominantes en el área monitoreada. La piñita $(M$. nudiflora) siendo la especie más importante en el área muestreada presenta un grado nocivo alto a medio. Además, tiene dos sistemas de propagación que le confieren características de maleza invasora del área, al igual que la lechosa (Euphorbia heterophylla L.), la ciperácea (Cyperus sp.) y la betilla (Ipomoea sp.), con menor importancia dentro del ecosistema monitoreado.

\section{CONCLUSIONES}

A pesar de la alta dominancia de especies, la diversidad y la riqueza de malezas fue relativamente alta.

El análisis funcional evidencia que las características reproductivas e interferencia de las especies de malezas encontradas en el cultivo determinan la importancia de cada especie dentro del área donde se establecen.

Las malezas con IVI dominantes fueron la piñita Murdannia nudiflora (L.) Brenan., y a la caminadora Rottboellia exaltata L.f., las cuales cubren entre el 5 y el $25 \%$ del área muestreada, siendo estas las principales malezas de la zona. 


\section{BIBLIOGRAFÍA}

Adkins, S. y Shabbir, A. (2014). Biology, ecology and management of the invasive parthenium weed (Parthenium hysterophorus L.). Pestic Manage Sci., 70 (7), 1023-1029. doi: 10.1002/ps.3708.

Bachega, L., Carvalho, B., Bianco, S. y Cecilio. F. (2013). Períodos de interferência de plantas daninhas na cultura do quiabo. Planta Daninha, 31 (1), 63-70. http://dx.doi.org/10.1590/S0100-83582013000100007

Bravo, N. (1991). Sobre la cuantificación de la diversidad ecológica. Hidrobiológica, 1 (1), 87-93. https://www. redalyc.org/articulo.oa? $\mathrm{id}=57801107$

Cardenas, J., Reyes, C. E., y Doll, J. D. (1972). Tropical weeds: Malezas tropicales. Bogotá, Colombia: Instituto Colombiano Agropecuario.

Cardina, J., Regnier, E. y Harrison, K. (1991). Long-Term Tillage Effects on Seed Banks in Three Ohio Soils. Weed Science, 39 (2), 186-194. Recuperado de http:// www.jstor.org/stable/4044914

Carvalho, B., Pitell, A., Cecílio, F., Bianco, C. y Guzzo, C. (2008). Interferência e estudo fitossociológico da comunidade infestante em beterraba de semeadura direta. Planta Daninha, 26 (3), 291-299. doi: 10.1590/ S0100-83582008000200005

Coelho, M., Bianco, S. y Carvalho, L. (2009). Interferência de plantas daninhas na cultura da cenoura (Daucus carota L.). Planta Daninha, 27 (Número Especial), 913-920. http://dx.doi.org/10.1590/S010083582009000500004.

Cordeau, S., Ryan, M., Bohan, D., Reboud, X. y Chauvel, B. (2017). Which Traits Allow Weed Species to Persist in Grass Margin Strips? Weed Science 201765 (3), 381-394. doi: 10.1017/wsc.2016.39

Cruz, D., Silva, G., Oliveira, F., Arcanjo, J. y Alves, J. (2009). Levantamento de plantas daninhas em área rotacionada com as culturas da soja, milho e arroz irrigado no cerrado de Roraima. Agroambiente, 3 (1), 58-63. doi: 10.18227/1982-8470ragro.v3i1.248

Curtis, J. y Mcintosh, R. (1951). An upland forest continuum in the prarie-forest border region of Wisconsin. Ecology, 32, 476-496. http://dx.doi. org/10.2307/1931725

Escobar, M. (2007). Efecto de comportamiento de la caminadora (Rottboellia exaltata L.f.) en el cultivo de maíz en la zona de Quevedo. Tesis no publicada. Universidad Técnica Estatal de Quevedo, Quevedo, Los Ríos, Ecuador.

Espinoza, G. (2008). Determinación de las principales malezas en potreros y su relación con las prácticas de manejo realizadas en las ganaderías bovinas de la Provincia de Los Ríos. Tesis no publicada, Escuela Superior Politécnica del Litoral, Guayaquil, Ecuador.
Gaba, S., Perronne, R., Fried, G., Gardarin, A., Bretagnolle, F., Biju-Duval, L., Colbach, N., Cordeau, S., FernándezAparicio, M., Gauvrit, C., Gibot-Leclerc, S Guillemin, J., Moreau, D., Munier-Jolain, N., Strbik, F. y Reboud, $X$. (2017). Response and effect traits of arable weeds in agro-ecosystems: a review of current knowled, ge. Weed Research.57, 123-147. doi: 10.1111/wre.12245

Gómez, A. y Rivera, H. (1987). Manejo y control integrado de malezas en plantaciones de café y potreros de la zona cafetera. Chinchiná, Colombia: Federación Nacional de Cafeteros

Hammer, Ø. (2001). Paleontological Statistics. Version 3.12. Natural History Museum. University of Oslo.

Instituto Nacional de Meteorología e Hidrología (2015). Anuarios Meteorológicos entregados por el Instituto Nacional de Meteorología e Hidrología (INAMHI) Quito, Ecuador. http://www.serviciometeorologico. gob.ec/boletines-climaticos-y-agricolas/

Johanns, O. y Contiero, R. (2006). Efeitos de diferentes períodos de controle e convivência de plantas daninhas com a cultura da mandioca. Ci. Agron., 37 (3), 326-331. http://ccarevista.ufc.br/seer/index.php/ ccarevista/article/view/175

Labrada, R., Caseley, J. y Parker C. (2004). Manejo de Malezas para Países en Desarrollo. Roma, Italia: FAO Producción y Protección Vegetal.

Lamprecht, H. 1990. Silvicultura en los trópicos. Los ecosistemas forestales en los bosques tropicales $y$ sus especies arbóreas: posibilidades y métodos para un aprovechamiento sostenido. Eschborn, Alemania: Instituto de Silvicultura de la Universidad de Göttingen.

Manpreer, S., Makhan, S. B. y Bhagirath, S. C. (2014). The critical period for weed control in dryseeded rice. Crop Prot., 66, 80-85. https://doi.org/10.1016/j. cropro.2014.08.009

Margalef, R. (1969). Diversity and stability: a practical proposal and a model of interdependence. Diversity and stability in ecological systems. Brookhaven symposia in Biology, 22, 25-37.

Marqués, P., Silva, M., López, S., Correa, P., Araujo, S., Costa, A. y Muniz, H. (2011). Dinâmica de populações e fitossociologia de plantas daninhas no cultivo do feijão-caupi e mandioca no sistema corte e queima com o uso de arado. Planta Daninha, 29 (Número Especial), 981-989. http://dx.doi.org/10.1590/S010083582011000500004

Matloob, A., Khaliq, A., Tanveer, A., Hussain, S., Aslam, F. y Singh, B. (2015). Weed dynamics as influenced by tillage system, sowing time and weed competition duration in dryseeded rice. Crop Prot., 71, 25-38. doi: 10.1016/j.cropro.2015.01.009

Mejia, J. 2010. Manual de malezas en cultivos de plantación. Synmalezas. Editorial Syngenta. 521 p 
Moreno, C. (2001). Métodos para medir la biodiversidad. Zaragoza, España: SEA, vol. 1. M\&T-Manuales y Tesis.

Mueller-Dombois, D. y Ellenberg, H. (1974) Aims and Methods of Vegetation Ecology. Nueva York, Estados Unidos: John Wiley and Sons.

Ordeñana, O. (1992). Malezas Rol-ecofisiologia-fisiología morfología y taxonomía. especies importantes en el Ecuador. Guayaquil, Ecuador: Grafimpac S. A. 338 p

Palacios, R. (2016). Diversificación del control de malezas con herbicidas de diferentes modos de acción para asegurar la sustentabilidad de los herbicidas disponibles. Tesis no publicada, Universidad Técnica Estatal de Quevedo, Quevedo, Los Ríos, Ecuador.

Perronne, R., Le Corre, V., Bretagnolle, V. y Gaba, S. (2015). Stochastic processes and crop types shape weed community assembly in arable fields. Journal of Vegetation Science, 26, 348-359. doi: 10.1111/ jvs.12238

Rodríguez, B. (2013). Evaluación de tres mezclas de herbicidas hormonales comerciales para el control de escoba (Sida rhombifolia) en pastos San Agustín (Stenotaphrum secundatum), cantón El Empalme, Provincia del Guayas. Tesis (Ing. Agrónomo) no publicada, Universidad Técnica Estatal de Quevedo, Quevedo, Los Ríos, Ecuador.

Toro, J. y Briones, J. (1984). Malezas predominantes en los cultivos de ciclo corto del Litoral. Portoviejo, Ecuador: Ramírez.

Tursun, N., Datta, A., Tuncel, E. y Knezevic, L. (2015). Nitrogen application influenced the critical period for weed control in cotton. Crop Prot., 74, 85-91.

Venegas, F. y Muñoz, R. (1984). Malezas tropicales del Litoral ecuatoriano. Comunicación Técnica N. 9. Estación Experimental Pichilingue. Ecuador.

Vera, R. (2008). Efecto de cuatro arreglos espaciales en sistemas de producción mixta cacao-plátano sobre la intensidad de escoba de bruja (Crinipellis perniciosa (Stahel) Singer) y sigatoka negra (Mycosphaerella fijiensis Morelet) durante el primer año de la fase de establecimiento. Tesis no publicada, Escuela Politécnica del Ejército, Santo Domingo, Ecuador.

Yirefu, F., Tana, T., Tafesse, A. y Zekarias, Y. (2012). Competitive ability of sugarcane (Saccharum officinarum L.) cultivars to weed interference in sugarcane plantations of Ethiopia. Crop Prot., 32, 13843. doi: 10.1016/j.cropro.2011.10.016 